\title{
Endoscopic Resection of Upper Gastrointestinal Subepithelial Tumours: Our Clinical Experience and Results
}

\author{
Mehmet Zeki Buldanlı', Oktay Yener ${ }^{2}$ \\ ${ }^{1}$ Department of General Surgery, University of Health Sciences, Gülhane Training \\ and Research Hospital, Ankara, Turkey; \\ ${ }^{2}$ Department of General Surgery, Istanbul Medeniyet University, Göztepe Training \\ and Research Hospital, Istanbul, Turkey
}

Received February 3, 2021; Accepted January 31, 2022.

Key words: Endoscopy - Subepithelial tumour - Upper gastrointestinal diseases Endoscopic submucosal dissection

\begin{abstract}
Upper gastrointestinal subepithelial tumours (SETs) are generally asymptomatic and clinically insignificant and have malign, borderline and benign variants. In advanced endoscopic procedures, histopathological diagnosis and endoscopic resection are possible and feasible. In this study, we examined our approach to upper gastrointestinal subepithelial tumours and our clinical results. Adult patients who applied to Surgical Endoscopy unit between January 2014 and January 2015 were included in the study. The patients' files and final histopathological diagnoses were recorded and analysed retrospectively for this single-center study. SET lesion lower than $30 \mathrm{~mm}$ and the lesion whose endoscopic submucosal dissection attemption was included in the study. The total of 8 patients were four female (50\%) and four male (50\%), aged 31-66 years (median, 53 years). The tumoral lesions were located 4 (50\%) patients in esophagus, 3 (37.5\%) patients in stomach and one (12.5\%) patient in duodenum and their diameter ranged from 5 to $30 \mathrm{~mm}$ (median, $14 \mathrm{~mm}$ ). Post-interventional no complications or abdominal symptoms were encountered. Also, in early follow-ups for six months, no recurrence was observed. Our experiences together with literature reported here, indicated endoscopic resection is a safe and effective method of treatment for most patients with upper gastrointestinal SETs.
\end{abstract}

Mailing Address: Dr. Oktay Yener, Department of General Surgery, Istanbul Medeniyet University, Göztepe Training and Research Hospital, Plaj Yolu Kaya apt. 23/10, Caddebostan, Istanbul, Turkey; e-mail oktayener@gmail.com 


\section{Introduction}

Submucosal masses or lesions often referred to as "submucosal tumours", represent a growth underneath the mucosa of the gastrointestinal (Gl) tract whose etiology cannot be determined by $\mathrm{Gl}$ endoscopy or barium contrast studies (Geis et al., 1996). It can also be detected with oral contrast-enhanced abdominal computed tomography (CT). However, the term "submucosal tumour" is inappropriate, because many of these lesions do not arise from the submucosa and many of them are not tumours (Halpin et al., 1993). Thus, "subepithelial" is a more appropriate term than "submucosal". Hence, other authors call these abnormalities subepithelial lesions, because they are covered by normal mucosa. These can be caused by external compression by the neighbouring organs or by intramural lesions. However, submucosal is still recognized and used (Kojima et al., 1999; Sakamoto et al., 2010).

Upper Gl subepithelial tumours (SETs) are generally asymptomatic and clinically insignificant and have malign, borderline and benign variants with various histopathological types. In advanced upper $\mathrm{Gl}$ endoscopic procedures, histopathological diagnosis and endoscopic resection are possible and feasible (Cho and Korean ESD Study Group, 2016). In this study, we examined our approach to upper gastrointestinal subepithelial tumours and our clinical results.

\section{Material and Methods}

Adult patients who applied to Istanbul Medeniyet University, Göztepe Training and Research Hospital, Department of General Surgery, Surgical Endoscopy unit between January 2014 and January 2015 were included in the study. The patients' files and final histopathological reports including diagnoses were recorded and analysed retrospectively for this single-center study. SET lesion lower than $30 \mathrm{~mm}$ and the lesion whose endoscopic submucosal dissection (ESD) attemption was included in the study.

Upper Gl endoscopy was planned for the patients with appropriate indications in line with their upper gastrointestinal system complaints. The procedure was planned after oral and intravenous contrast-enhanced abdominal CT. Submucosal lesion of the stomach was found by upper gastrointestinal endoscopy performed. Complete blood count (CBC), coagulation and routine biochemistry blood tests were performed in all patients before the procedure. All patients were referred for pre-interventional anesthesia examination. Acetyl salicylic acid and/or other anticoagulant drugs were withdrawn before intervention. Patients informed consent was taken before endoscopic procedures. After 8 hours of fasting, the procedures were performed by the same endoscopy team in a single center. Surgeon as an endoscopist, endoscopy technician, surgery resident, anesthesiologist and anesthesia technician participated in the procedures. Vital signs of the patients were monitored during the procedure. Sedation was performed with topical anesthetic drugs and intravenous propofol and managed by the anesthesiologist. 
The procedure was started with standard gastroscope after the sedation. Gastric residuals were cleaned by gastric irrigation and aspiration with $0.9 \%$ normal saline solution for proper visualization and also detecting the lesion with margins. Endoscopic submucosal dissection was carried out as follows; special solution ( $0.4 \%$ hyaluronic acid, $2 \mathrm{ml}$ indigo carmine, $1 / 10.000$ epinephrine, $0.9 \%$ normal saline solution) was injected by endoscopic injector into the submucosal layer for lifting the lesion at the marked margin of resection, moving from a distal to a proximal direction, and the lesion was elevated. Circumferential incision was performed by endoscopic knife and endoscopic cautery for the en bloc excision from surrounding tissue. The submucosal lesion was dissected using an endoscopic snare and an endoscopic cutting knife. Then the tumour was resected from the surrounding tissues with negative surgical margins. If needed, endoscopic cautery and/or clips were used for hemostasis after resection. The specimen was extracted by endoscopic snare or endoscopic grasper and sent to the medical pathology department for histopathological examination.

If there was any concern about the complications (bleeding, perforation or anesthetic complications) post-procedurally, the patients were hospitalized and followed-up closely.

This study complied with the World Medical Association Declaration of Helsinki. Statistically, median and percentage values calculated with Microsoft Office Excel ${ }^{\circledR}$ program.

\section{Results}

The total of 8 patients were four female (50\%) and four male (50\%), aged 31-66 years (median, 53 years). The tumoral lesions were located 4 (50\%) patients in the distal esophagus, 3 (37.5\%) patients in fundus part of stomach and one (12.5\%) patient in bulbus duodeni and their diameter ranged from 5 to $30 \mathrm{~mm}$ (median, $14 \mathrm{~mm}$ ) (Table 1). They were diagnosed as SETs from the upper $\mathrm{Gl}$ endoscopic appearance. Final histopathological diagnoses were benign leiomyoma in 5 (62.5\%) patients (Figure 1). The other diagnoses were gastrointestinal mesenchymal tumour, lipoma and gastrointestinal stromal tumour (GIST) (Table 1). When histopathological evaluation is made according to localization; three leiomyomas were in distal esophagus and two leiomyomas were in stomach. Also, one GIST was in stomach, one lipoma was in distal esophagus and one gastrointestinal mesenchymal tumour was in bulbus duodeni. When the pathology reports were examined, it was determined that all specimens were resected as the R0 resection and surgical margins were clear.

Leiomyomas were detected microscopically as smooth muscle tumour with minimal atypia and stained positively by smooth muscle actin and desmin. GIST was detected as sclerosing spindle subtype with bland spindle cells and stained positively c-KIT (CD117) and DOG-1. Lipoma was detected as mature homogeneous adipose tissue without atypia. Gastrointestinal mesenchymal tumour detected as an 


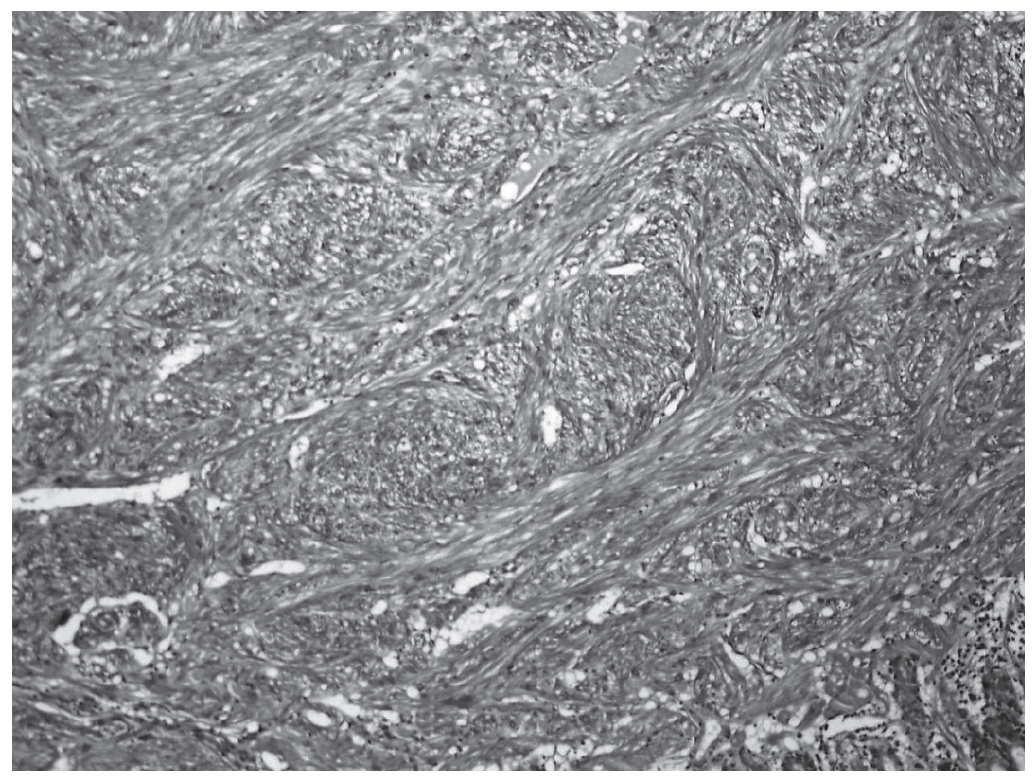

Figure 1 - Histological appearance of the benign subepithelial lesion (stained with hematoxylin-eosin, $\times 10$ ).

\section{Table 1 - Demographic and tumour characteristics of patients}

\begin{tabular}{lc}
\hline Age (year) & $31-66(53)$ \\
\hline Gender, male/female, $\mathrm{n}(\%)$ & $4 / 4(50 \% / 50 \%)$ \\
\hline Tumour size $(\mathrm{mm})$ & $5-30(14)$ \\
\hline Tumour localization, $\mathrm{n}(\%)$ & $4(50.0 \%)$ \\
Esophagus & $3(37.5 \%)$ \\
Stomach & $1(12.5 \%)$ \\
Duodenum & \\
\hline Final histopathological diagnosis of tumour, $\mathrm{n}(\%)$ & $5(62.5 \%)$ \\
Leiomyom & $1(12.5 \%)$ \\
GIST & $1(12.5 \%)$ \\
Gastrointestinal mesenchymal tumour & $1(12.5 \%)$ \\
Lipoma &
\end{tabular}

Values are median or $\mathrm{n}(\%)$; GIST - gastrointestinal stromal tumour

inflammatory myofibroblastic tumour with myofibroblastic spindle cell proliferation and mixed inflammation microscopically. It was stained by vimentin and ALK-1 positively.

Post-interventional no complications or abdominal symptoms were encountered. After the procedure, the patients were informed in detail about the process. Oral 
intake was started in four hour later without any trouble. If there was a concern about complications oral intake delayed. Patients were discharged with oral proton pump inhibitor and sucralfate medical treatment, recommendations and follow-up planning. The first control examination was scheduled for 15 days. In this way, patients were also informed about their histopathological findings. Subsequent follow-up examinations were scheduled at the first month, third month, and sixth month. In the third and sixth months, endoscopic evaluation controls were also performed, no pathology was observed. By the way, in follow-ups for six months, no recurrence or complications were observed.

\section{Discussion}

The majority of subepithelial tumours do not cause symptoms and are discovered incidentally during endoscopic or radiologic examinations. The overlying mucosa usually appears smooth and normal at endoscopy. If symptoms do occur, they are nonspecific such as abdominal pain, obstruction, hemorrhage and intussusceptions (Sakamoto et al., 2010; Inoue et al., 2012). Large submucosal neoplasms may outgrow their blood supply, ulcerate through the mucosa, and present as $\mathrm{Gl}$ bleeding. Firm subepithelial tumours may also present with obstructive symptoms, especially if they are located near the cardia or the pylorus. Subepithelial tumours obstructing the major or minor papilla may cause jaundice or pancreatitis. Pain and weight loss, often associated with large submucosal GISTs, are symptoms that suggest malignancy (Iwahashi et al., 2006). In this study, our patients presented with dyspeptic complaints and upper abdominal pain.

Park et al. (2004) have recently reported the experience of endoscopic enucleation of submucosal tumours of the esophagus and the stomach in 15 patients. There were four patients with GISTs of the stomach. Tumour tissue was removed completely in these patients but a small perforation occurred in one patient, in whom the tumour involved the muscularis propria and had grown outward to the serosa. In this study, the esophageal and gastric procedure performed to seven patients and no early complications were observed in these patients.

Rosch et al. (2004) have treated 14 patients with submucosal tumours of the esophagus and the stomach by endoscopic surgery. In their series, there were five patients with GIST of the stomach. No serious complications were encountered in these patients but complete removal was uncertain in two patients with gastric GISTs that originated in the muscularis propria. In this study, no complications or early recurrence were observed after the procedure. Also, all specimens were resected as the $\mathrm{R} 0$ resection and surgical margins were clear.

In a series of 51 patients by Chiu et al. (2019), the importance of location and size for the SET intervention was emphasized. Especially, per oral endoscopic tumour resection (POAT) was found safe for esophageal and gastric SET located at cardia, lesser curvature, and antrum. Also, they limited the tumour size $<40 \mathrm{~mm}$. In this study, tumour localizations and sizes are similar. POAT as an advanced minimal 
invasive technique, was not experienced in our center. So, we could not perform this procedure on patients with similar characteristics.

Endoscopic ultrasonography (EUS) can give findings in the differential diagnosis of SETs and in determining which tunica layer originates from. In addition, histopathological sampling can be done before the procedure with endoscopic ultrasound-guided fine needle aspiration and biopsy. For this reason, it can be considered in experienced centers together with interventional endoscopic procedures (Kim et al., 2019). In this study, CT imaging and routine endoscopy were performed pre-interventional and the patients were informed about not performing EUS and their consent was obtained.

With the development of endoscopic techniques and minimal invasive procedures, surgical procedures for intervention in SETs leave their place to endoscopic interventions. Endoscopic submucosal dissection (ESD), endoscopic enucleation, endoscopic excavation, endoscopic submucosal tunnel dissection, submucosal tunnel endoscopic resection, and endoscopic full-thickness resection (EFTR) are options for this treatment. Also, after these procedures endoscopic clipping can be performed because of the risk for bleeding (Kim and Kim, 2018). In this study, routine ESD procedure was applied after lifting and no problems were encountered during the procedure.

Kim et al. (2011) reported that the major complications of the ESD are bleeding and perforation. Perforation was stated with a rate of $4 \%$ in the first studies about this topic, but it is stated that this rate is decreasing with increasing experience. Bleeding was obtained with rates from 13 to $38 \%$. Stenosis, transient bacteremia, aspiration pneumonia, thromboembolism and anesthetic complications are other complications that were specified in the literature (Saito et al., 2014). In this study, per-interventional minor bleedings were encountered and immediate successful hemostasis performed with the endoscopic cautery and/or clips. Perforation and other complications were not observed.

In the literature, it was observed that follow-up was ranged from 6.1 months to 19.1 months after endoscopic procedures. These follow-ups were made with endoscopy and examinations. No recurrence was observed among the patients with complete resection in included studies (Kim and Kim, 2016). In this study, the patients were followed-up for six months and recurrence was not observed as well.

The limitations of this study are the low number of patients, not performing of other advanced endoscopic interventions and procedures for this study, the short post-interventional follow-up period with results and retrospective design of study.

\section{Conclusion}

Consequently, our experiences together with literature reported here, indicated endoscopic resection is a safe and effective method of treatment for most patients with upper gastrointestinal SETs. However, prospective randomized studies and reviews involving more patient populations are needed for this subject. 


\section{References}

Chiu, P. W. Y., Yip, H. C., Teoh, A. Y. B., Wong, V. W. Y., Chan, S. M., Wong, S. K. H., Ng, E. K. W. (2019) Per oral endoscopic tumor (POET) resection for treatment of upper gastrointestinal subepithelial tumors. Surg. Endosc. 33(4), 1326-1333.

Cho, J. W., Korean ESD Study Group (2016) Current guidelines in the management of upper gastrointestinal subepithelial tumors. Clin. Endosc. 49(3), 235-240.

Geis, W., Baxt, R., Kim, H. C. (1996) Benign gastric tumors. Minimally invasive approach. Surg. Endosc. 10(4), 407-410.

Halpin, R., Thomson, S., Catterall, N., Haffejee, A. (1993) Smooth muscle tumors of the stomach: Clinicopathological aspects. J. R. Coll. Surg. Edinb. 38(1), 23-27.

Inoue, H., Ikeda, H., Hosoya, T., Onimaru, M., Yoshida, A., Eleftheriadis, N., Maselli, R., Kudo, S. (2012) Submucosal endoscopic tumor resection for subepithelial tumors in the esophagus and cardia. Endoscopy 44(3), 225-230.

Iwahashi, M., Takifuji, K., Ojima, T., Nakamura, M., Nakamori, M., Nakatani, Y., Ueda, K., Ishida, K., Naka, T., Ono, K., Yamaue, H. (2006) Surgical management of small gastrointestinal stromal tumors of the stomach. World J. Surg. 30(1), 28-35.

Kim, S. G., Song, J. H., Hwang, J. H. (2019) Current status of endoscopic ultrasonography in gastrointestinal subepithelial tumors. Clin. Endosc. 52(4), 301-305.

Kim, S. H., Moon, J. S., Youn, Y. H., Lee, K. M., Lee, S. J. (2011) Management of the complications of endoscopic submucosal dissection. World J. Gastroenterol. 17(31), 3575-3579.

Kim, S. Y., Kim, K. O. (2016) Management of gastric subepithelial tumors: The role of endoscopy. World J. Gastrointest. Endosc. 8(11), 418-424.

Kim, S. Y., Kim, K. O. (2018) Endoscopic treatment of subepithelial tumors. Clin. Endosc. 51(1), 19-27.

Kojima, T., Takahashi, H., Parra-Blanco, A., Kohsen, K., Fujita, R. (1999) Diagnosis of submucosal tumor of the upper $\mathrm{Gl}$ tract by endoscopic resection. Gastrointest. Endosc. 50(4), 516-522.

Park, Y. S., Park, S. W., Kim, T. I., Song, S. Y., Choi, E. H., Chung, J. B., Kang, J. K. (2004) Endoscopic enucleation of upper-Gl submucosal tumors by using an insulated-tip electrosurgical knife. Gastrointest. Endosc. 59(3), 409-415.

Rosch, T., Sarbia, M., Schumacher, B., Deinert, K., Frimberger, E., Toermer, T., Stolte, M., Neuhaus, H. (2004) Attempted endoscopic en bloc resection of mucosal and submucosal tumors using insulated-tip knives: a pilot series. Endoscopy 36(9), 788-801.

Saito, I., Tsuji, Y., Sakaguchi, Y., Niimi, K., Ono, S., Kodashima, S., Yamamichi, N., Fujishiro, M., Koike, K. (2014) Complications related to gastric endoscopic submucosal dissection and their managements. Clin. Endosc. 47(5), 398-403.

Sakamoto, H., Kitano, M., Kudo, M. (2010) Diagnosis of subepithelial tumors in the upper gastrointestinal tract by endoscopic ultrasonography. World J. Radiol. 2(8), 289-297. 